\title{
Patency of anterior circulation branch vessels after Pipeline embolization: longer-term results from 82 aneurysm cases
}

\author{
Leonardo Rangel-Castilla, MD, ${ }^{1,4}$ Stephan A. Munich, MD, ${ }^{1,4}$ Naser Jaleel, MD, PhD, ${ }^{1,4}$ \\ Marshall C. Cress, MD, ${ }^{1,4}$ Chandan Krishna, MD, ${ }^{1,4}$ Ashish Sonig, MD, MS, MCh, ${ }^{1,4}$ \\ Kenneth V. Snyder, MD, PhD, ${ }^{1-4}$ Adnan H. Siddiqui, MD, PhD, ${ }^{1,2,4-6}$ and Elad I. Levy, MD, MBA ${ }^{1,2,4,5}$ \\ Departments of ${ }^{1}$ Neurosurgery, ${ }^{2}$ Radiology, and ${ }^{3}$ Neurology, Jacobs School of Medicine and Biomedical Sciences, University at \\ Buffalo, State University of New York; ${ }^{4}$ Department of Neurosurgery, Gates Vascular Institute at Kaleida Health; ${ }^{5}$ Toshiba Stroke \\ and Vascular Research Center, University at Buffalo, State University of New York; and ${ }^{6}$ Jacobs Institute, Buffalo, New York
}

OBJECTIVE The Pipeline Embolization Device (PED) has become increasingly used for the treatment of intracranial aneurysms. Given its high metal surface area coverage, there is concern for the patency of branch vessels that become covered by the device. Limited data exist regarding the patency of branch vessels adjacent to aneurysms that are covered by PEDs. The authors assessed the rate of intracranial internal carotid artery, anterior circulation branch vessel patency following PED placement at their institution.

METHODS The authors retrospectively reviewed the records of 82 patients who underwent PED treatment between 2009 and 2014 and in whom the PED was identified to cover branch vessels. Patency of the anterior cerebral, posterior communicating, anterior choroidal, and ophthalmic arteries was evaluated using digital subtraction angiography preoperatively and postoperatively after PED deployment and at longer-term follow-up.

RESULTS Of the 127 arterial branches covered by PEDs, there were no immediate postoperative occlusions. At angiographic follow-up (mean 10 months, range 3-34.7 months), arterial side branches were occluded in 13 (15.8\%) of 82 aneurysm cases and included 2 anterior cerebral arteries, 8 ophthalmic arteries, and 3 posterior communicating arteries. No cases of anterior choroidal artery occlusion were observed. Patients with branch occlusion did not experience any neurological symptoms.

CONCLUSIONS In this large series, the longer-term rate of radiographic side branch arterial occlusion after coverage by a flow diverter was $15.8 \%$. Terminal branch vessels, such as the anterior choroidal artery, remained patent in this series. The authors' series suggests that branch vessel occlusions are clinically silent and should not deter aneurysm treatment with flow diversion.

https://thejns.org/doi/abs/10.3171/2016.4.JNS16147

KEY WORDS artery branch; endovascular; flow diversion; intracranial aneurysm; patency; Pipeline device; vascular disorders

$\mathrm{F}$ LOW diversion is increasingly recognized as an important tool in the treatment of intracranial aneurysms. $^{3-9,12}$ The Pipeline Embolization Device (PED, Covidien) is a flow-diversion device made of $25 \%$ platinum tungsten and $75 \%$ cobalt chromium. ${ }^{6,8}$ Flow diverters disrupt flow into the aneurysm sac while maintaining normal blood flow through parent and branch vessels. Disrupted flow into the aneurysm causes flow stagnation, thrombosis, and sealing of the ostium of the aneurysm through endothelialization and neointimal proliferation across the device struts. There are theoretical concerns that this flow stagnation may also occur in branch vessels covered by the PED, particularly in small perforating arteries, resulting in branch vessel thrombosis. This prompted the FDA to approve the PED for use only in the internal carotid artery (ICA) from the petrous to the superior hy-

ABBREVIATIONS ACA = anterior cerebral artery; $\mathrm{AChA}$ = anterior choroidal artery; DSA = digital subtraction angiography; ICA = internal carotid artery; MRA = MR angiography; OphA = ophthalmic artery; PCOA = posterior communicating artery; PED = Pipeline Embolization Device.

SUBMITTED January 19, 2016. ACCEPTED April 11, 2016.

INCLUDE WHEN CITING Published online June 10, 2016; DOI: 10.3171/2016.4.JNS16147. 
pophyseal segments. Similarly, concern for branch vessel integrity has resulted in widespread reluctance toward PED use in the posterior circulation where small perforating arteries serve critical brainstem structures. ${ }^{9}$

The rate and clinical consequence of branch vessel occlusion after treatment with PEDs remains poorly defined. Recently, authors of small patient series have sought to elucidate this widespread concern, evaluating the rates of ophthalmic artery (OphA), posterior communicating artery $(\mathrm{PCoA})$, and anterior choroidal artery (AChA) integrity following PED placement. ${ }^{2,3,10,14}$ In this study, we evaluated the rate of branch vessel patency following PED placement in aneurysms in 82 patients treated at our institution.

\section{Methods}

The protocol for this study had the approval of the University at Buffalo institutional review board. Medical records from consecutive patients with anterior circulation aneurysms treated with the PED between 2009 and 2014 were reviewed retrospectively from a prospectively maintained database to obtain demographic data (including age, sex, and medical comorbidities) as well as clinical data regarding complications and outcome. Two investigators who were not involved in the procedures independently reviewed all preprocedure and postprocedure angiographic data, including aneurysm location, number of PEDs deployed, and patency of anterior circulation branch vessels (i.e., OphA, PCoA, AChA, and anterior cerebral artery [ACA]). In situations in which the 2 investigators disagreed on aspects of the angiographic data, discrepancies were reconciled in a blinded fashion by a third investigator.

Our protocol for PED deployment has been described previously. ${ }^{7}$ Briefly, patients were pretreated with aspirin (325 mg) and clopidogrel $(75 \mathrm{mg}$ ) for 5 days prior to treatment. If this was not possible, they were given loading doses (aspirin $650 \mathrm{mg}$ and clopidogrel $600 \mathrm{mg}$ ) prior to their procedure. Therapeutic levels were determined using platelet inhibition assays (P2Y12) and aspirin responses (VerifyNow Assay System, Accumetrics, Inc.). Systemic anticoagulation was maintained during the procedure (activated clotting factor $>250$ seconds). The patients were subsequently maintained on a regimen of aspirin therapy for life and clopidogrel for at least 6 months. All procedures were performed under conscious sedation and with the use of local anesthetics. All PEDs were deployed using a bi- or triaxial system. A 0.027 -inch microcatheter was used to obtain distal access past the segment of the vessel harboring the target aneurysm. The PED was sized to match the maximum diameter of the target vessel. Control digital subtraction angiography (DSA) was performed immediately following PED placement. Follow-up DSA was performed between 3 and 12 months postprocedure. If aneurysm occlusion was confirmed, further follow-up consisted of MRA and was conducted on a yearly basis thereafter. If aneurysm occlusion was not confirmed, yearly DSA was performed until occlusion was confirmed.

\section{Labeling and Indications}

According to the US FDA, the PED (study device) is indicated for the endovascular treatment of adults $(\geq 22$ years of age) with large or giant wide-necked intracranial aneurysms in the ICA from the petrous to the superior hypophyseal segments.

\section{Results}

We identified 120 patients who were treated with the PED for aneurysms in the anterior circulation between 2009 and 2014 at our institution. Thirty-eight patients were excluded from analysis for reasons of no branch vessel coverage seen on angiography $(\mathrm{n}=21)$ and lack of follow-up angiography $(\mathrm{n}=17)$. Therefore, we included 82 patients with 82 aneurysms in our subsequent analyses (Table 1). Baseline characteristics for these patients including demographics and aneurysm characteristics are presented in Table 2. The mean aneurysm size was $11.3 \pm$ $6.7 \mathrm{~mm}$. The ophthalmic segment was the most frequent location for aneurysms in our series.

A total of 127 instances of branch vessel coverage were identified in 82 patients with 82 aneurysms (Table 3). This included 76 OphAs, 28 PCoAs, 21 AChAs, and 2 ACAs. There was no evidence of branch vessel occlusion immediately after PED deployment. On follow-up angiography (mean 10.0 months, range 3-34.7 months), we identified 13 branch vessel (OphA, PCoA, and ACA) occlusions. The OphA was occluded in 8 of 76 instances (10.5\%) (Fig. 1), the PCoA was occluded in 3 of 28 instances (10.7\%) (Fig. 2), and the ACA in 2 of 2 instances (Fig. 3). No instance of AChA occlusion was observed on follow-up angiography. Subgroup analysis of PCoA vessel patency showed no occlusion when the PCoA was fetal-type (0 of 4 instances) (Table 4). We found a relationship between the number of PEDs deployed and the percentage of branch occlusion (Table 4). We observed no adverse clinical sequelae in patients who experienced branch vessel occlusion.

Additionally, in an effort to identify factors that correlated with branch vessel occlusion, we analyzed several additional variables in patients with OphA occlusion (Table 5) and those with PCoA or ACA occlusion (Table 6)

TABLE 1. Patient demographics*

\begin{tabular}{lc}
\hline \multicolumn{1}{c}{ Characteristic } & Value \\
\hline Patients analyzed & 120 \\
\hline Included in analysis & 82 \\
\hline Mean age in yrs & $58.6 \pm 11.7$ \\
\hline Sex & $5(6.1)$ \\
\hline Male & $77(93.9)$ \\
\hline Female & $34(41.5)$ \\
\hline No. of medical comorbidities & $2(2.4)$ \\
\hline HTN & $4(4.9)$ \\
\hline CAD & $5(6.1)$ \\
\hline DM & $49(59.8)$ \\
\hline Stroke & \\
\hline Smoking & \\
\hline CAD $=$ coronary artery disease; DM $=$ diabetes mellitus; HTN $=$ hypertension. \\
* Values represent the number of patients (\%) unless specified otherwise. The \\
mean value is presented as the mean \pm SD.
\end{tabular}


TABLE 2. Aneurysm characteristics

\begin{tabular}{cc}
\hline \multicolumn{1}{c}{ Variable } & Value \\
\hline Mean size in $\mathrm{mm}$ & $11.3 \pm 6.7$ \\
\hline Location $(\mathrm{n})$ & \\
\hline Cavernous & 14 \\
\hline Superior hypophyseal & 12 \\
\hline OphA & 20 \\
\hline PCoA & 11 \\
\hline Paraclinoid & 10 \\
\hline Supraclinoid & 4 \\
\hline Other & 11 \\
\hline Ruptured $(\mathrm{n})$ & 11 \\
\hline
\end{tabular}

after PED placement, including aneurysm size and location, number of PEDs used, and use of adjunctive coiling. We found a relationship between the number of PEDs used and the occurrence of branch vessel occlusion (Table $5)$ : multiple PEDs were used in 7 of $8(88 \%)$ patients who developed OphA occlusion. Patients with PCoA and ACA occlusion had only 1 PED device placed.

\section{Discussion}

Flow diversion is becoming an increasingly used strategy for the treatment of intracranial aneurysms. Flowdiversion devices are supposed to maintain blood flow through parent and branch vessels while disrupting flow into the aneurysm sac. There are concerns regarding possible occlusion of side branches and perforating vessels that unavoidably would be covered by the PED. In our study of 82 patients with 82 intracranial aneurysms treated with PED and 127 instances of branch vessel coverage, we found $13(15.8 \%)$ cases of branch vessel occlusion that included the OphA, PCoA, and ACA. None of these vessel occlusions occurred during or immediately after PED deployment. All occlusions were clinically asymptomatic and were found only on follow-up angiograms. The overall incidence of side branch occlusion has been reported to be between $2.3 \%$ and $21 \%$. $^{3,4,10,12,14,15}$

\section{Ophthalmic Artery}

Of the 76 OphAs covered by $\mathrm{PED}(\mathrm{s})$ immediately after PED deployment, we found $8(10.5 \%)$ instances of arterial occlusion over time (Table 5 and Fig. 1). None of the 8

TABLE 3. Patency of ICA branch vessels after PED placement*

\begin{tabular}{|c|c|c|c|c|c|}
\hline \multirow[b]{2}{*}{ Vessel } & \multirow{2}{*}{$\begin{array}{c}\text { No. of } \\
\text { Vessels }\end{array}$} & \multicolumn{2}{|c|}{ Immediate } & \multicolumn{2}{|c|}{ Follow-Up } \\
\hline & & Patent & Occluded & Patent & Occluded \\
\hline OphA & 76 & 76 (100) & 0 & $68(89.4)$ & $8(10.5)$ \\
\hline PCoA & 28 & $28(100)$ & 0 & $25(89.3)$ & $3(10.7)$ \\
\hline Adult & 24 & $24(100)$ & 0 & $21(88)$ & $3(13)$ \\
\hline Fetal & 4 & $4(100)$ & 0 & $4(100)$ & $0(0)$ \\
\hline AChA & 21 & 21 & 0 & $21(100)$ & $0(0)$ \\
\hline $\mathrm{ACA}$ & 2 & 2 & 0 & $0(0)$ & $2(100)$ \\
\hline
\end{tabular}

* Values indicate the number of instances of branch vessel coverage (\%).

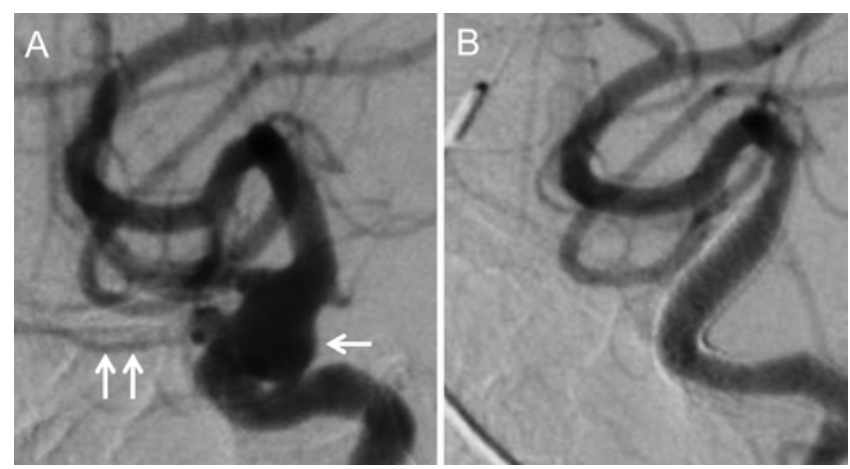

FIG. 1. Occlusion of the OphA after treatment with the PED. A: Pretreatment lateral digital subtraction angiogram showing a paraclinoid ICA aneurysm (arrow). Notice the presence of the OphA (double arrow). The patient underwent endovascular treatment with the PED. B: Lateral follow-up DS angiogram obtained at the 6-month follow-up, demonstrating complete obliteration of the paraclinoid aneurysm and absence of the OphA.

patients experienced visual loss. Among these instances, 4 of the patients had 3 PEDs placed, 3 patients had 2 PEDs placed, and only 1 patient had 1 PED placed, suggesting that the number of PEDs used could contribute to branch artery occlusion. Other than the number of PEDs, there were no other risk factors that appeared to increase the risk of OphA branch occlusion. Other variables, such as the presence of the OphA originating from the aneurysm sac, immediate posttreatment intraaneurysmal flow stasis, or patient age, were not significantly correlated. Puffer et al. ${ }^{10}$ found similar results when assessing the patency of the OphA in 20 patients after flow-diversion treatment for paraclinoid aneurysms. They found a $21 \%$ incidence of OphA occlusion with proximal thrombosis when the OphA was covered with flow-diversion devices. However, their study was limited by the small number of aneurysms treated. Similar to our findings, those events were well tolerated clinically. The OphA has collateral anastomoses with branches of the external carotid artery that could contribute to proximal OphA occlusion if the PED decreases the flow and distal anastomosis takes over endorgan arterial supply. Unlike Puffer et al., we found that
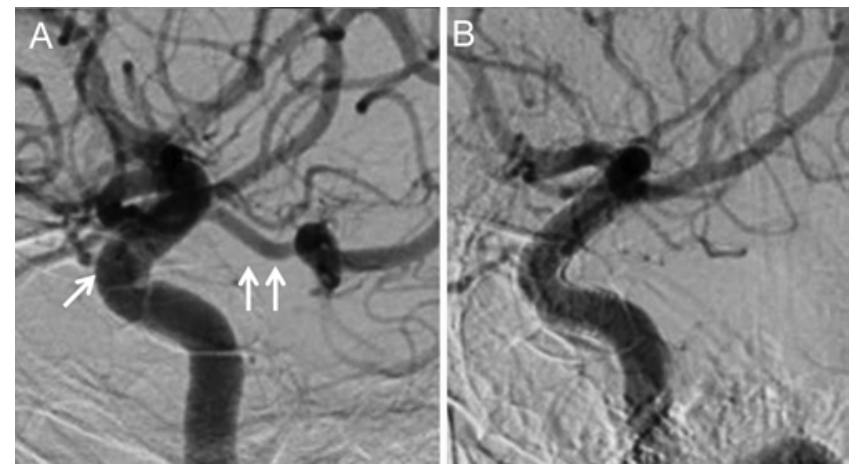

FIG. 2. Occlusion of the PCoA after PED placement. A: Pretreatment lateral DSA showing a superior hypophyseal artery aneurysm (double density) (arrow). Observe the presence of the PCoA (double arrow). The patient underwent endovascular treatment with the PED. B: An 8-month follow-up lateral DSA demonstrating complete obliteration of the aneurysm and absence of the PCoA. 

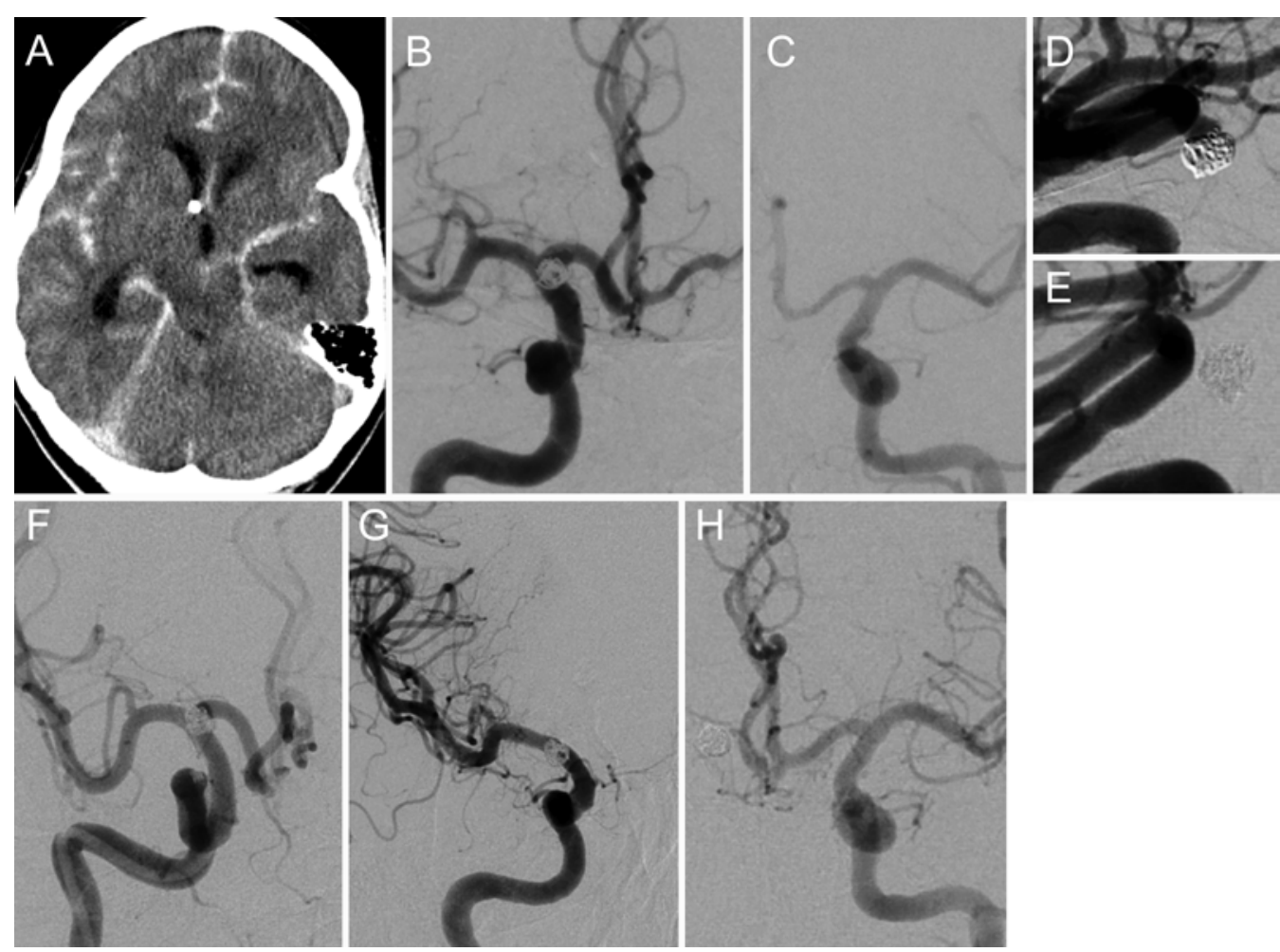

FIG. 3. Occlusion of the ACA after PED placement. This 47-year-old woman presented with a ruptured aneurysm and subarachnoid hemorrhage. A: CT scan showing diffuse subarachnoid hemorrhage. B: Anteroposterior projection DS angiogram demonstrating that the right ICA aneurysm is successfully coiled. Notice the filling of the right and left ACAs from the ipsilateral ICA. C: Anteroposterior projection DS angiogram of the left ICA showing filling of the left ACA only. D: A 2-year follow-up DS angiogram showing aneurysm neck recurrence, which was treated with a PED. E: A 7-month follow-up DS angiogram demonstrating complete aneurysm obliteration. F: Anteroposterior projection, right ICA, DS angiogram obtained immediately after PED deployment, showing a patent right ACA with filling of the anterior communicating artery and left ACA. G and H: Seven-month DS angiograms after PED deployment demonstrating occlusion of the right $A C A(G)$ and now filling of both ACAs by the left ICA $(H)$.

the majority of our patients ( 7 of 8 ) had 2 or 3 overlapping PED devices. Computational fluid dynamics modeling has shown that after placement of a flow diverter that covers up to $90 \%$ of the perforating vessel ostium, flow is reduced only by less than $10 \%$ of baseline. ${ }^{1}$ Four other studies have shown rates of OphA occlusion after PED deployment that range from $0 \%$ to $21 \% .^{10,12,14,15}$ It would have been interesting to investigate OphA collaterals from the external carotid artery circulation. As the patients remained clinically asymptomatic, one can conclude that there is collateralization.

\section{Posterior Communicating and Anterior Cerebral Arteries}

Among the 28 PCoAs covered by PED(s), we observed

\section{TABLE 4. Aneurysm treatment}

\begin{tabular}{ccc}
\hline Variable & No. of Patients (\%) & Occlusion at Follow-Up (\%) \\
\hline No. of PEDs & & \\
\hline 1 & $46(56.1)$ & $7(15.2)$ \\
\hline 2 & $24(29.3)$ & $6(25)$ \\
\hline 3 & $11(13.4)$ & $4(36.4)$ \\
\hline 4 & $1(1.2)$ & $0(0)$ \\
\hline Coiling & $16(19.5)$ & \\
\hline
\end{tabular}

$3(10.7 \%)$ instances of arterial occlusion at longer-term follow-up (Fig. 2). All PCoAs were covered by only a single PED device. The 3 patients were asymptomatic (Table 6). None of them had decreased flow or occlusion immediately after the procedure. All occlusions were seen at follow-up angiography. Of the 11 cases of the PCoA being covered by a PED, Brinjikji et al. ${ }^{3}$ found 5 cases (45\%) in which the PCoA was occluded or flow was diminished at a mean follow-up of 12.6 months. None of these patients had clinical symptoms. Vedantam et al. ${ }^{14}$ found 1 (7.1\%) PCoA occlusion among 11 arteries covered by a PED. In our series, in all 3 instances of PCoA occlusion, the PCoAs were nonfetal-type. Unfortunately, none of the 3 patients had an injection of the vertebral artery to assess for possible retrograde filling of the PCoA.

Regarding the ACA, we found 2 instances of arterial occlusion after PED deployment at follow-up. One patient had a ruptured right ICA bifurcation aneurysm treated with primary coiling. Aneurysm neck recurrence was observed on the 2-year follow-up angiogram. The decision was made to treat the aneurysm recurrence with flow diversion. Because of the aneurysm location, the ACA had to be jailed by the flow diverter. At follow-up angiography performed 10 months after PED treatment, we found that the $A_{2}$ artery was not filling from the ipsilateral ACA but from the contralateral ACA through a well-developed an- 
TABLE 5. Features of treatment and clinical outcome in patients with OphA occlusion after PED placement

\begin{tabular}{|c|c|c|c|c|c|c|c|}
\hline $\begin{array}{c}\text { Patient } \\
\text { No. }\end{array}$ & Age (yrs), Sex & Location (artery) & Size $(\mathrm{mm})$ & No. of PEDs & Adjunctive Coiling? & Follow-Up (mos) & Clinical Sequelae \\
\hline 1 & $44, \mathrm{~F}$ & Rt OphA & 10.3 & 1 & Yes & 3.0 & None \\
\hline 2 & $41, \mathrm{~F}$ & Lt superior hypophyseal & 10 & 2 & No & 4.3 & None \\
\hline 3 & $55, \mathrm{~F}$ & Lt paraophthalmic & 10.6 & 2 & No & 3.5 & None \\
\hline 4 & $62, \mathrm{~F}$ & Rt OphA & 13 & 2 & No & 7.5 & None \\
\hline 5 & $59, \mathrm{~F}$ & Rt cavernous ICA & 20 & 3 & No & 15.9 & None \\
\hline 6 & $53, F$ & Rt paraclinoid & 6 & 3 & No & 9.6 & None \\
\hline 7 & $50, \mathrm{~F}$ & Rt OphA & 6 & 3 & Yes & 3.3 & None \\
\hline 8 & $63, \mathrm{~F}$ & Rt paraclinoid & 21 & 3 & No & 34.7 & None \\
\hline
\end{tabular}

terior communicating artery (Fig. 3). The second instance was in a patient with a recurrent large PCoA aneurysm that was treated originally with primary coiling technique. The recurrent aneurysm was treated with flow diversion; similarly, the ACA had to be jailed by the flow diverter. At 7.5 months follow-up after PED treatment, the $A_{2}$ was found not to be filling from the ipsilateral ACA but from the contralateral ACA. To the best of our knowledge, there are no previous reports of this phenomenon. Flow diverters placed across the origin of small perforating vessels may maintain flow through the ostium due to a pressure gradient; the same effect may not be true for large collateralrich vessels like the PCOA or ACA. The pressure gradient across the device struts is not enough to maintain flow due to the opposing effect of the collateral flow, which creates a neutral gradient and angiographic occlusion. This observed phenomenon prompts considerations that we should keep in mind when treating aneurysms with flow diverters close to and incorporating large branches. Retrograde flow can also maintain flow into the aneurysm, resulting in no aneurysm occlusion. In these situations, other endovascular techniques, such as stent-assisted coiling or flow diversion with coiling, should be considered.

\section{Anterior Choroidal Artery}

Of the 21 AChAs covered by a PED, we did not observe any instance of occlusion (Fig. 3). Three studies have shown AChA occlusion rates between $0 \%$ and $7 \%$ after flow diversion with PEDs. ${ }^{2,14,15}$ Among 14 patients with the AChA covered by a PED, Brinjikji et al. ${ }^{2}$ found 1 occlusion $(6.7 \%)$ at the time of 6-month follow-up angiography. The patient did not show signs or symptoms of neurological deterioration. As is the case with OphA and PCOA occlusion after PED treatment, collateral flow determines the potential for and clinical significance of vessel occlusion. The lateral posterior choroidal artery, posterior cerebral artery, and PCoA contribute collateral flow to the AChA territory. These collaterals have been observed in cases of chronic ICA occlusion. ${ }^{13}$

In normal circumstances, terminal arteries without significant collateral supply, such as the AChA, are more likely to remain patent after coverage by a flow diverter. The postulated theory is that the pressure gradient across end arteries is more than that across vessels with a rich collateral supply, thus increasing the threshold for branch occlusion by a flow diverter. Similar to other studies, ${ }^{10,12,14,15}$ our results support this theory. Ischemic stroke rates after PED deployment approach 2\%,11,12 and ischemic strokes are more common when giant and posterior circulation aneurysms are treated. These strokes are rarely related to major side branch occlusion. Neurological deficits due to ischemic events are more common within the first 30 days after the procedure. ${ }^{4}$ In the current series, none of the patients had any neurological deficits, echoing previous studies findings. Other than multiple PED usage, we were unable to identify any particular risk factors associated with side branch occlusion.

\section{Study Limitations}

This study has the inherent limitations of a retrospective study. Patients do not have follow-up MRI to evaluate for clinically silent infarctions potentially related to a branch occlusion. No posterior circulation aneurysms are included.

\section{Conclusions}

Results of this large series demonstrated a $15.8 \%$ rate of radiographic side branch arterial occlusion after cover-

TABLE 6. Features of treatment and clinical outcome in patients with PCoA and ACA occlusion after PED placement

\begin{tabular}{|c|c|c|c|c|c|c|c|}
\hline $\begin{array}{c}\text { Patient } \\
\text { No. }\end{array}$ & Age (yrs), Sex & Location (artery) & Branch Occluded, Size (mm) & No. of PEDs & Coiling? & Follow-Up (mos) & Clinical Sequelae \\
\hline 1 & $49, F$ & Lt supraclinoid ICA & $\mathrm{PCoA}, 2^{*}$ & 1 & No & 5.8 & None \\
\hline 2 & $60, F$ & Rt PCoA & PCoA, 10 & 1 & No & 3.3 & None \\
\hline 3 & $49, \mathrm{M}$ & Rt superior hypophyseal & PCoA, $1.6^{*}$ & 1 & No & 6.6 & None \\
\hline 4 & $30, F$ & Lt superior hypophyseal & $\mathrm{ACA}, 6$ & 1 & Yes & 8.4 & None \\
\hline 5 & $62, F$ & Lt PCoA & ACA, 5 & 1 & Yes & 7.5 & None \\
\hline
\end{tabular}

* Blister type. 
age by a flow diverter. These branches included the ACA, OphA, and PCoA. There were no AChA occlusions. These instances were not associated with any new neurological deficits. The number of PEDs placed seems to correlate with an increased risk of branch occlusion. Branch coverage of richly collateralized vessels is clinically well tolerated.

\section{Acknowledgments}

We thank Paul H. Dressel, BFA, for preparation of the illustrations and Debra J. Zimmer for editorial assistance.

\section{References}

1. Appanaboyina S, Mut F, Lohner R, Scrivano E, Miranda C, Lylyk P, et al: Computational modelling of blood flow in side arterial branches after stenting of cerebral aneurysms. Int J Comput Fluid Dyn 22:669-676, 2008

2. Brinjikji W, Kallmes DF, Cloft HJ, Lanzino G: Patency of the anterior choroidal artery after flow-diversion treatment of internal carotid artery aneurysms. AJNR Am J Neuroradiol 36:537-541, 2015

3. Brinjikji W, Lanzino G, Cloft HJ, Kallmes DF: Patency of the posterior communicating artery after flow diversion treatment of internal carotid artery aneurysms. Clin Neurol Neurosurg 120:84-88, 2014

4. Brinjikji W, Murad MH, Lanzino G, Cloft HJ, Kallmes DF: Endovascular treatment of intracranial aneurysms with flow diverters: a meta-analysis. Stroke 44:442-447, 2013

5. Fiorella D, Lylyk P, Szikora I, Kelly ME, Albuquerque FC, McDougall CG, et al: Curative cerebrovascular reconstruction with the Pipeline embolization device: the emergence of definitive endovascular therapy for intracranial aneurysms. J Neurointerv Surg 1:56-65, 2009

6. Fiorella D, Woo HH, Albuquerque FC, Nelson PK: Definitive reconstruction of circumferential, fusiform intracranial aneurysms with the pipeline embolization device. Neurosurgery 62:1115-1121, 2008

7. Kan P, Siddiqui AH, Veznedaroglu E, Liebman KM, Binning MJ, Dumont TM, et al: Early postmarket results after treatment of intracranial aneurysms with the pipeline embolization device: a U.S. multicenter experience. Neurosurgery 71:1080-1088, 2012

8. Lylyk P, Miranda C, Ceratto R, Ferrario A, Scrivano E, Luna $\mathrm{HR}$, et al: Curative endovascular reconstruction of cerebral aneurysms with the Pipeline embolization device: the Buenos Aires experience. Neurosurgery 64:632-643, N6, 2009

9. Nelson PK, Lylyk P, Szikora I, Wetzel SG, Wanke I, Fiorella D: The pipeline embolization device for the intracranial treatment of aneurysms trial. AJNR Am J Neuroradiol 32:34-40, 2011

10. Puffer RC, Kallmes DF, Cloft HJ, Lanzino G: Patency of the ophthalmic artery after flow diversion treatment of paraclinoid aneurysms. J Neurosurg 116:892-896, 2012

11. Saatci I, Yavuz K, Ozer C, Geyik S, Cekirge HS: Treatment of intracranial aneurysms using the pipeline flow-diverter embolization device: a single-center experience with longterm follow-up results. AJNR Am J Neuroradiol 33:14361446, 2012

12. Szikora I, Berentei Z, Kulcsar Z, Marosfoi M, Vajda ZS, Lee $\mathrm{W}$, et al: Treatment of intracranial aneurysms by functional reconstruction of the parent artery: the Budapest experience with the pipeline embolization device. AJNR Am J Neuroradiol 31:1139-1147, 2010

13. Takahashi S, Tobita M, Takahashi A, Sakamoto K: Retrograde filling of the anterior choroidal artery: vertebral angiographic sign of obstruction in the carotid system. Neuroradiology 34:504-507, 1992
14. Vedantam A, Rao VY, Shaltoni HM, Mawad ME: Incidence and clinical implications of carotid branch occlusion following treatment of internal carotid artery aneurysms with the pipeline embolization device. Neurosurgery 76:173-178, 2015

15. Yu SC, Kwok CK, Cheng PW, Chan KY, Lau SS, Lui WM, et al: Intracranial aneurysms: midterm outcome of pipeline embolization device-a prospective study in 143 patients with 178 aneurysms. Radiology 265:893-901, 2012

\section{Disclosures}

The authors report the following. Dr. Cress: consultant for Toshiba Medical Research Institute USA. Dr. Snyder: Consultant for Toshiba, ev3, Abbott Vascular, Medtronic, and Boston Scientific. Dr. Siddiqui: financial interest in Buffalo Technology Partners Inc., Cardinal, International Medical Distribution Partners, Medina Medical Systems, Neuro technology Investors, StimSox, and Valor Medical; consultant for Amnis Therapeutics Ltd., Cerebrotech Medical Systems Inc., CereVasc LLC, Codman, Corindus Inc., Covidien (acquired by Medtronic), GuidePoint Global Consulting, Lazarus (acquired by Medtronic), Medina Medical (acquired by Medtronic), Medtronic, MicroVention, Neuravi, Penumbra, Pulsar Vascular, Rapid Medical, Rebound Medical, Reverse Medical (acquired by Medtronic), Silk Road Medical Inc., Stryker, The Stroke Project Inc., Three Rivers Medical Inc., and W.L. Gore \& Associates; principal investigator/national steering committee for Covidien SWIFT PRIME Trial, LARGE Trial, Medtronic SWIFT DIRECT, MicroVention CONFIDENCE Study, MicroVention FRED Trial, Penumbra 3D Separator Trial, Penumbra COMPASS Trial, Penumbra INVEST Trial, and POSITIVE Trial; and board member for Intersocietal Accreditation Committee. Dr. Levy: ownership in Intratech Medical Ltd., Blockade Medical LLC, NeXtGen Biologics; consultant for Blockade Medical LLS and Pulsar; national principal investigator for Covidien SWIFT Prime Trials; honoraria for training and lectures from Covidien and Abbott; and member of the advisory board for Stryker, NeXtGen, and MEDX.

\section{Author Contributions}

Conception and design: Rangel-Castilla, Munich, Jaleel. Acquisition of data: all authors. Analysis and interpretation of data: all authors. Drafting the article: Rangel-Castilla, Munich, Jaleel. Critically revising the article: all authors. Reviewed submitted version of manuscript: all authors. Statistical analysis: RangelCastilla, Munich, Jaleel.

\section{Supplemental Information}

\section{Previous Presentations}

Portions of this work were presented in abstract form at the 83rd AANS Annual Scientific Meeting, May 2-6, 2015, Washington, DC.

\section{Current Affiliations}

Dr. Rangel-Castilla: Department of Neurosurgery, Wayne State University, Detroit, MI.

Dr. Cress: Department of Neurosurgery, University of Florida, Orlando, FL.

Dr. Krishna: Department of Neurosurgery, Mayo Clinic, Scottsdale, AZ.

\section{Correspondence}

Elad I. Levy, Department of Neurosurgery, University at Buffalo, 100 High St., Ste. B4, Buffalo, NY 14203. email: elevy@ubns. com. 